applying Theorem 1 , that $\left|\phi_{1}(t)-\phi_{2}(t)\right| \leqq \chi(t)$ for $t \geqq \tau$ where $\chi(t)$ is the maximal solution of $z^{\prime}=\omega(t, z)+\epsilon$ through $(\tau, \delta)$. Further the first few examples of page 37 of [4] can all be solved by the application of Corollary 1. Similarly Theorems 1 and 2 can be used in a natural way to extend the results of [3] concerning bounds on the norm of a solution of a differential equation.

\title{
REFERENCES
}

1. R. Bellman, Stability theory of differential equations, McGraw-Hill, London, 1953.

2. I. Bihari, $A$ generalisation of a lemma of Bellman and its application to uniqueness problems of differential equations, Acta Math. Acad. Sci. Hungar. 7 (1956), 81-94.

3. C. E. Langenhop, Bounds on the norm of a solution of a general differential equation, Proc. Amer. Math. Soc. 11 (1960), 795-799.

4. E. A. Coddington and Norman Levinson, Theory of ordinary differential equations, McGraw-Hill, New York, 1955, pp. 8-9.

OSMANIA UNIVERSITY, Hyderabad, INDIA

\section{THE G-FUNCTIONS AS UNSYMMETRICAL FOURIER KERNELS. II}

\section{ROOP NARAIN}

1. A function $K(x)$ by means of which an arbitrary function $f(x)$ subject to appropriate conditions, is capable of being represented as a repeated integral of the form

$$
f(x)=\int_{0}^{\infty} K(x u) \int_{0}^{\infty} K(u y) f(y) d y d u
$$

has been called a Fourier kernel by Hardy and Titchmarsh [1, p. 116]. This is a symmetrical formula. There are also unsymmetrical formulae of the type

$$
f(x)=\int_{0}^{\infty} K(x u) \int_{0}^{\infty} H(u y) f(y) d y d u
$$

in which the kernels in the two integrals are different functions. If $f(t)$ is not continuous at $t=x, f(x)$ on the left-hand side of (1.1) or (1.2) is replaced by

$$
\frac{1}{2}\{f(x+0)+f(x-0)\} .
$$

Received by the editors January 6, 1962 . 
The functions $K(x)$ and $H(x)$ have been referred to as $a$ pair of unsymmetrical Fourier kernels. Examples of such kernels have been given by various authors from time to time. In a recent paper [2, p. 950] the author obtained formally a pair of unsymmetrical kernels . in terms of Meijer's $G$-function. These functions [2, p. 953] are

$$
\begin{aligned}
& K(x)=2 \gamma x^{\gamma-1 / 2} G_{p+q, m+n}^{m, p}\left(x^{2 \gamma} \mid \begin{array}{l}
a_{1}, \cdots, a_{p}, b_{1}, \cdots, b_{q} \\
c_{1}, \cdots, c_{m}, d_{1}, \cdots, d_{n}
\end{array}\right), \\
& H(x)=2 \gamma x^{\gamma-1 / 2} G_{p+q, m+n}^{n, q}\left(x^{2 \gamma} \mid \begin{array}{l}
-b_{1}, \cdots,-b_{q},-a_{1}, \cdots,-a_{p} \\
-d_{1}, \cdots,-d_{n},-c_{1}, \cdots,-c_{m}
\end{array}\right),
\end{aligned}
$$

where $\gamma>0, n-p=m-q>0$ and

$$
\sum_{j=1}^{p} a_{j}+\sum_{j=1}^{q} b_{j}=\sum_{j=1}^{m} c_{j}+\sum_{j=1}^{n} d_{j}
$$

The importance of these functions is due to their very general yet simple form from which many known as well as new kernels can be deduced as special cases $[2, £ 3]$.

The $G$-function is a sum of hypergeometric functions each of which is usually an entire function. It is defined $[3$, p. 207] by the integral

$$
\begin{aligned}
G_{p, q}^{m, n}\left(x \mid \begin{array}{l}
a_{1}, \cdots, a_{p} \\
b_{1}, \cdots, b_{q}
\end{array}\right) & \\
& =\frac{1}{2 \pi i} \int_{L} \frac{\prod_{j=1}^{m} \Gamma\left(b_{j}+s\right) \prod_{j=1}^{n} \Gamma\left(1-a_{j}-s\right)}{\prod_{j=m+1}^{q} \Gamma\left(1-b_{j}-s\right) \prod_{j=n+1}^{p} \Gamma\left(a_{j}+s\right)} x^{-s} d s,
\end{aligned}
$$

where $m, n, p$ and $q$ are integers with

$$
q \geqq p+1, \quad 0 \leqq n \leqq p, \quad 0 \leqq m \leqq q .
$$

The poles of the integrand must be simple and those of $\Gamma\left(b_{j}+s\right)$, $j=1, \cdots, m$, must lie on one side of the contour $L$ and those of $\Gamma\left(1-a_{j}-s\right), j=1, \cdots, n$, must lie on the other side.

The case of symmetrical formula in which $K(x)=H(x)=$ a certain $G$-function has been considered by Fox $[4$, p. 395$]$. In the present paper which is in continuation of the paper [2] we shall formulate convergence conditions with which the functions $K(x)$ and $H(x)$ of (1.3) and (1.4) give rise to the formula (1.2). The work of this paper is based upon a theorem of Hardy and Titchmarsh [1, p. 148, Theorem 4] which will be reproduced here in the form in which it is going to be used. 
2. Let $k(s)$ and $h(s)$ be the Mellin transforms of $K(x)$ and $H(x)$ respectively, i.e.,

$$
k(s)=\int_{0}^{\infty} K(x) x^{s-1} d x, \quad h(s)=\int_{0}^{\infty} H(x) x^{s-1} d x,
$$

where $s=\sigma+i t, \sigma$ and $t$ real. According to Hardy and Titchmarsh a function $k(s)$ will be said to belong to $k^{\prime}, k(s) \in k^{\prime}$, if it satisfies the following conditions.

(a) $k(s)$ is regular in a strip $\sigma_{1}<\sigma<\sigma_{2}$ where $\sigma_{1}<0, \sigma_{2}>1$ except possibly for a finite number of simple poles on the imaginary axis, and

(b)

$$
k(s)=\left\{\alpha+\frac{\beta}{s}+O\left(\frac{1}{|s|^{2}}\right)\right\} \Gamma(s) \operatorname{Cos}\left(\frac{1}{2} s \pi\right) \quad \text { for } t=+\infty,
$$

$$
k(s)=\left\{\alpha^{\prime}+\frac{\beta^{\prime}}{s}+O\left(\frac{1}{|s|^{2}}\right)\right\} \Gamma(s) \operatorname{Cos}\left(\frac{1}{2} s \pi\right) \quad \text { for } t=-\infty,
$$

where $\alpha, \beta, \alpha^{\prime}, \beta^{\prime}$ are complex numbers.

The following theorem due to Hardy and Titchmarsh will be of use in the present work.

THEOREM. If

(i) $k(s) \in k^{\prime}$,

(ii) $h(s) \in k^{\prime}$,

(iii) $k(s)$ and $h(s)$ satisfy the functional relation

$$
k(s) h(1-s)=1,
$$
then

$$
\int_{0}^{\infty} K(x u) \int_{0}^{\infty} H(u y) f(y) d y d u=\frac{1}{2}\{f(x+0)+f(x-0)\}
$$

3. We now proceed to establish the relation (2.3) for the case of functions

$$
K(x)=K(x), \quad H(x)=H(x) .
$$

We shall prove the following theorem.

THEOREM 1. If

(i)

$$
\gamma>0, \quad 0<n-p=m-q=\eta / 2, \text { say, }
$$


(ii)

$$
\begin{aligned}
& \sum_{j=1}^{p} a_{j}+\sum_{j=1}^{q} b_{j}=\sum_{j=1}^{m} c_{j}+\sum_{j=1}^{n} d_{j}, \\
& \operatorname{Rl}\left(\frac{1}{2}-a_{j}\right)>\frac{1}{2 \eta}, \quad j=1, \cdots, p, \\
& \operatorname{Rl}\left(\frac{1}{2}+b_{j}\right)>\frac{1}{2 \eta}, \quad j=1, \cdots, q, \\
& \operatorname{Rl}\left(\frac{1}{2}+c_{j}\right) \geqq \frac{1}{2 \eta}, \quad j=1, \cdots, m, \\
& \operatorname{Rl}\left(\frac{1}{2}-d_{j}\right) \geqq \frac{1}{2 \eta}, \quad j=1, \cdots, n,
\end{aligned}
$$

(iii)

(iv) $y^{\eta / \gamma-1 / 2} f(y) \in L(0, \infty)$ and $f(y)$ is of bounded variation near $y=x(x>0)$, then

$$
\int_{0}^{\infty} K(x u) \int_{0}^{\infty} H(u y) f(y) d y d u=\frac{1}{2}\{f(x+0)+f(x-0)\},
$$

where $K(x)$ and $H(x)$ are given by (1.3) and (1.4), respectively.

Proof. We first make the following transformations of variables in (3.1),

$$
x=X^{\eta / 2 \gamma}, \quad y=Y^{\eta / 2 \gamma}, \quad u=(U / \eta)^{\eta / 2 \gamma} .
$$

By an easy computation and adjustments, (3.1) can be written as

$$
\begin{aligned}
\int_{0}^{\infty} K^{*}(X U) & \int_{0}^{\infty} H^{*}(U Y) f^{*}(Y) d Y d U \\
= & \frac{1}{2}\left\{f^{*}(X+0)+f^{*}(X-0)\right\},
\end{aligned}
$$

where

$$
\begin{aligned}
& K^{*}(x)=\frac{\eta^{1-\eta / 4 \gamma}}{2 \gamma} K\left(\left\{\frac{x}{\eta}\right\}^{\eta / 2 \gamma}\right) x^{\eta / 4 \gamma-1 / 2}, \\
& H^{*}(x)=\frac{\eta^{1-\eta / 4 \gamma}}{2 \gamma} H\left(\left\{\frac{x}{\eta}\right\}^{\eta / 2 \gamma}\right) x^{\eta / 4 \gamma-1 / 2},
\end{aligned}
$$

and $f^{*}(x)=x^{\eta / 4 \gamma-1 / 2} f\left(x^{\eta / 2 \gamma}\right)$. We have then to deal with the kernels $K^{*}(x)$ and $H^{*}(x)$ instead of $K(x)$ and $H(x)$. The reasons for making these transformations will appear in the next section.

Using the definition (1.5) of the $G$-function, $K^{*}(x)$ is given by 


$$
\begin{aligned}
K^{*}(x) & =\sqrt{\eta}\left(\frac{x}{\eta}\right)^{(\eta-1) / 2} G_{p+q, m+n}^{m, p}\left(\left(\frac{x}{\eta}\right)^{\eta} \mid \begin{array}{l}
a_{1}, \cdots, a_{p}, b_{1}, \cdots, b_{q} \\
c_{1}, \cdots, c_{m}, d_{1}, \cdots, d_{n}
\end{array}\right) \\
& =\frac{1}{2 \pi i} \int_{L} \sqrt{\eta} \frac{\prod_{j=1}^{m} \Gamma\left(c_{j}+s\right) \prod_{j=1}^{p} \Gamma\left(1-a_{j}-s\right)}{\prod_{j=1}^{n} \Gamma\left(1-d_{j}-s\right) \prod_{j=1}^{q} \Gamma\left(b_{j}+s\right)}\left(\frac{x}{\eta}\right)^{-\eta++(\eta-1) / 2} d s
\end{aligned}
$$

where the contour $L$ of integration is a straight line parallel to the imaginary axis in the $s$-plane separating the poles of $\Gamma\left(c_{j}+s\right)$, $j=1, \cdots, m$, and $\Gamma\left(1-a_{j}-s\right), j=1, \cdots, p$.

Making one more transformation $\eta s-(\eta-1) / 2=S$, the above equation becomes

$$
K^{*}(x)=\frac{1}{2 \pi i} \int_{L} \mathscr{K}(S) x^{-S} d S,
$$

where

$$
\Re(S)=\frac{\prod_{j=1}^{m} \Gamma\left(1 / 2+c_{j}-1 / 2 \eta+S / \eta\right)}{\prod_{j=1}^{n} \Gamma\left(1 / 2-d_{j}+1 / 2 \eta-S / \eta\right)}
$$

$$
\times \frac{\prod_{j=1}^{p} \Gamma\left(1 / 2-a_{j}+1 / 2 \eta-S / \eta\right)}{\prod_{j=1}^{q} \Gamma\left(1 / 2+b_{j}-1 / 2 \eta+S / \eta\right)} \eta^{s-1 / 2}
$$

and the contour $L$ is any straight line parallel to the imaginary axis in the $S(=\sigma+i t)$ plane for which $0<\sigma<1 / 2$. Evidently $\mathcal{K}(S)$ is the Mellin transform of $K^{*}(x)$ in the sense inverse to (2.1).

If $\operatorname{sC}(S)$ is the Mellin transform of $H^{*}(x)$, it can be seen in a similar manner that

$$
\begin{gathered}
\operatorname{HC}(S)=\frac{\prod_{j=1}^{n} \Gamma\left(1 / 2-d_{j}-1 / 2 \eta+S / \eta\right)}{\prod_{j=1}^{m} \Gamma\left(1 / 2+c_{j}+1 / 2 \eta-S / \eta\right)} \\
\times \frac{\prod_{j=1}^{q} \Gamma\left(1 / 2+b_{j}+1 / 2 \eta-S / \eta\right)}{\prod_{j=1}^{p} \Gamma\left(1 / 2-a_{j}-1 / 2 \eta+S / \eta\right)} \eta^{s-1 / 2} .
\end{gathered}
$$


We shall now use the theorem of $\$ 2$ to establish (3.3). It will be shown that $\mathfrak{K}(S)$ and $\mathfrak{H C}(S)$ satisfy conditions (i), (ii) and (iii) and $f^{*}(Y)$ satisfies condition (iv) of that theorem.

Let $\Sigma$ denote the strip $0 \leqq \sigma \leqq 1$ and $-\infty<t<\infty$, where $S=\sigma+i t$. From (3.7), the poles of $\mathcal{K}(S)$ are at the points

$$
\begin{aligned}
-\eta r-\eta\left(c_{j}+\frac{1}{2}-\frac{1}{2 \eta}\right), & j=1, \cdots, m, \\
\eta r-\eta\left(a_{j}-\frac{1}{2}-\frac{1}{2 \eta}\right), & j=1, \cdots, p,
\end{aligned}
$$

and from (3.8), those of $3 C(S)$ are at the points

$$
\begin{aligned}
-\eta r+\eta\left(d_{j}-\frac{1}{2}+\frac{1}{2 \eta}\right), & j=1, \cdots, n, \\
\eta r+\eta\left(b_{j}+\frac{1}{2}+\frac{1}{2 \eta}\right), & j=1, \cdots, q,
\end{aligned}
$$

where $r$ is a positive integer or zero. From conditions (iii) of Theorem 1 , it follows that if only the inequalities hold, then all the $a_{j}$ poles of $\mathscr{K}(S)$ and $b_{j}$ poles of $\mathscr{H}(S)$ lie to the right of $\Sigma$ while $c_{j}$ poles of $\mathscr{K}(S)$ and $d_{j}$ poles of $\operatorname{FC}(S)$ lie to the left of $\Sigma$, none of them being on the boundaries of $\Sigma$. If, however, the equality holds for some of the $c_{j}$ 's and $d_{j}$ 's in (iii), then there are simple poles of $\mathcal{K}(S)$ and $\mathcal{H}(S)$ on the imaginary axis and there can be at the most $m$ of $\mathcal{K}(S)$ and $n$ of $\operatorname{HC}(S)$. Thus since the only singularities of $\mathscr{K}(S)$ and $\mathcal{H}(S)$ are isolated simple poles it follows that $\sigma_{1}<0$ and $\sigma_{2}>1$ can be found such that $\Re(S)$ and $\Re(S)$ are both regular in the strip $\sigma_{1}<\sigma<\sigma_{2}$ except possibly for a finite number of simple poles on the imaginary axis.

To prove that $\mathscr{K}(S)$ and $\mathcal{H}(S)$ also satisfy the second requirement for being of class $k^{\prime}$ we shall find the asymptotic expansions of $\Re(S)$ and $\operatorname{sC}(S)$ for large positive and negative $t$.

The asymptotic expansion [3, p. 48] of the Gamma function for large $s$ is given by

$$
\begin{aligned}
\log \Gamma(s+a)= & \left(s+a-\frac{1}{2}\right) \log s-s+\frac{1}{2} \log (2 \pi) \\
& +\frac{B}{s}+O\left(\frac{1}{|s|^{2}}\right),
\end{aligned}
$$

where $|\arg s|<\pi, B$ is a constant independent of $s$.

In the expression for $\mathfrak{K}(S)$ let the Gamma functions involving 
$-S / \eta$ be changed into the Gamma functions containing $+S / \eta$ by the formula $[3$, p. 3]

$$
\Gamma(z) \Gamma(1-z)=\pi / \operatorname{Sin} \pi z
$$

so that we get

$$
\begin{gathered}
\nVdash(S)=\frac{\prod_{j=1}^{m} \Gamma\left(1 / 2+c_{j}-1 / 2 \eta+S / \eta\right) \prod_{j=1}^{n} \Gamma\left(1 / 2+d_{j}-1 / 2 \eta+S / \eta\right)}{\prod_{j=1}^{p} \Gamma\left(1 / 2+a_{j}-1 / 2 \eta+S / \eta\right) \prod_{j=1}^{q} \Gamma\left(1 / 2+b_{j}-1 / 2 \eta+S / \eta\right)} \\
\times \frac{\prod_{j=1}^{n} \operatorname{Sin} \pi\left(1 / 2-d_{j}+1 / 2 \eta-S / \eta\right)}{\prod_{j=1}^{p} \operatorname{Sin} \pi\left(1 / 2-a_{j}+1 / 2 \eta-S / \eta\right)} \pi^{p-\eta} \eta^{S-1 / 2}
\end{gathered}
$$

Using (3.9), we notice that for large $S,|\arg S|<\pi$, the contribution to the asymptotic expansion of $\Re(S)$ due to the factors involving Gamma functions only is

$$
\begin{aligned}
& \exp \left[\left(\sum_{j=1}^{m} c_{j}\right.\right.\left.+\sum_{j=1}^{n} d_{j}-\sum_{j=1}^{p} a_{j}-\sum_{j=1}^{q} b_{j}\right) \log \frac{S}{\eta} \\
&\left.+\frac{1}{\eta}(m+n-p-q)\left\{\left(S-\frac{1}{2}\right) \log \frac{S}{\eta}-S\right\}\right]\left[A_{1}+\frac{B_{1}}{S}+O\left(\frac{1}{|S|^{2}}\right)\right] \\
&=\exp \left[\left(S-\frac{1}{2}\right) \log \frac{S}{\eta}-S\right]\left[A_{1}+\frac{B_{1}}{S}+O\left(\frac{1}{|S|^{2}}\right)\right],
\end{aligned}
$$

on using conditions (i) and (ii) of the theorem,

$$
=\eta^{1 / 2-S} \exp \left[\left(S-\frac{1}{2}\right) \log S-S\right]\left[A_{1}+\frac{B_{1}}{S}+O\left(\frac{1}{|S|^{2}}\right)\right],
$$

where $A_{1}$ and $B_{1}$ are constants independent of $S$. Using (3.9) again, this expression is seen to be equivalent to

$$
\eta^{1 / 2-S} \Gamma(S)\left\{A_{2}+\frac{B_{2}}{S}+O\left(\frac{1}{|S|^{2}}\right)\right\},
$$

where $A_{2}$ and $B_{2}$ are constants.

Next we consider the contribution to the asymptotic expansion of $\varkappa(S)$ due to the factors involving Sine functions. On allowing $|t|$ to become large, it is easily seen that 


$$
\frac{\operatorname{Sin} \pi(\theta-S / \eta)}{(\operatorname{Cos}(S \pi / 2))^{2 / \eta}}=D_{1}+O\left(e^{-|t|}\right),
$$

where $\theta$ and $\eta$ are independent of $S$ and $D_{1}$ is a constant such that and

$$
D_{1}=2^{2 / \eta-1} \exp \{\pi i(\theta-1 / 2)\} \quad \text { for large positive } t
$$

$$
D_{1}=2^{2 / \eta-1} \exp \{-\pi i(\theta-1 / 2)\} \quad \text { for large negative } t .
$$

There are $n$ Sine factors in the numerator and $p$ in the denominator of $\mathcal{K}(S)$ and since $n-p=\eta / 2$, the total contribution of all the Sine factors to the asymptotic expansion of $\Re(S)$ is a factor of the form

$$
\left\{D_{2}+O\left(e^{-|t|}\right)\right\} \operatorname{Cos}(S \pi / 2),
$$

where $D_{2}$ is a constant such that

$$
\begin{aligned}
& D_{2}=2^{1-\eta / 2} \exp \left\{\pi i\left(\frac{1}{4}-\sum_{j=1}^{n} d_{j}+\sum_{j=1}^{p} a_{j}\right)\right\} \quad \text { for large positive } t \\
& D_{2}=2^{1-\eta / 2} \exp \left\{-\pi i\left(\frac{1}{4}-\sum_{j=1}^{n} d_{j}+\sum_{j=1}^{p} a_{j}\right)\right\} \quad \text { for large negative } t .
\end{aligned}
$$

The asymptotic expansion of $\Re(S)$ can now be written by taking the product of (3.11), (3.12) and the factor $\pi^{\eta / 2} \eta^{S-1 / 2}$. We thus have finally the following result. For $S=\sigma+i t$ and sufficiently large values of $|t|$ the asymptotic expansion of $\Re(S)$ is given by

$$
\mathcal{K}(S) \sim \Gamma(S) \operatorname{Cos}(S \pi / 2)\left\{\alpha+\frac{\beta}{S}+O\left(\frac{1}{|S|^{2}}\right)\right\},
$$

where the constants $\alpha$ and $\beta$ each have one value for large positive $t$ and another for large negative $t$.

In an exactly similar manner it is seen that for large values of $|t|$,

$$
\mathfrak{H}(S) \sim \Gamma(S) \operatorname{Cos}(S \pi / 2)\left\{\alpha^{\prime}+\frac{\beta^{\prime}}{S}+O\left(\frac{1}{|S|^{2}}\right)\right\},
$$

where $\alpha^{\prime}$ and $\beta^{\prime}$ are constants each having one value when $t$ is large and positive and another when $t$ is large and negative.

We have thus shown that $\mathscr{K}(S)$ and $\mathcal{H}(S)$ both belong to the class $\boldsymbol{k}^{\prime}$ so that conditions (i) and (ii) of the theorem of $\$ 2$ are fulfilled. The third condition of the theorem is that

$$
\mathfrak{H}(S) \mathfrak{H C}(1-S)=1
$$

and this is obviously true from (3.7) and (3.8). 
The fourth condition is that $f^{*}(Y)$ belongs to $L(0, \infty)$ and that it is of bounded variation near $Y=X(X>0)$. This demands that

$$
\int_{0}^{\infty}\left|Y^{\eta / 4 \gamma-1 / 2} f\left(Y^{\eta / 2 \gamma}\right)\right| d Y
$$

must exist and the integrand must be of bounded variation near $Y=X(X>0)$. On putting $Y^{\eta / 2 \gamma}=y$, it follows from (iv), in the enunciation of Theorem 1 , that these two requirements are fulfilled.

All the conditions of the theorem of Hardy and Titchmarsh have thus been shown to be satisfied. The conclusion of that theorem must therefore follow and consequently the equation (3.3) is true. On using the transformations (3.2) in the reverse sense, (3.3) implies the truth of (3.1).

4. Here we shall obtain the asymptotic expansions of $K(x)$ and $H(x)$ which will explain the necessity of effecting the transformations (3.2) in the proof of Theorem 1.

The classical Fourier kernels such as $\operatorname{Sin} x, \operatorname{Cos} x$, and $\sqrt{x} J_{\nu}(x)$ all behave in a very similar manner as $x \rightarrow \infty$. When $x$ is complex they all tend to infinity with exponential rapidity and when $x$ is real they all oscillate finitely as $x \rightarrow \infty$ just like $\operatorname{Cos} x$. It is in fact the behaviour of a function on the real axis which decides whether it is a Fourier kernel or not. The functions $K(x)$ and $H(x)$ of (1.3) and (1.4) do not, however, necessarily oscillate finitely as $x \rightarrow \infty$ through positive values. But the kernels $K^{*}(x)$ and $H^{*}(x)$ in the transformed integral equation (3.3) do oscillate in the familiar manner like $\operatorname{Cos} x$ as $x \rightarrow \infty$ through positive values as is shown below. It is for this reason that the equation (3.1) is first transformed by the relations (3.2) into (3.3) and then the theorem of Hardy and Titchmarsh is applied to it.

The asymptotic expansion of $K(x)$ as defined by (1.3) is obtained from the work of Meijer [5, p. 1065, Theorem 18, assertion 4]. For large real positive $x$, we have

$$
\begin{aligned}
& K(x) \sim x^{\gamma / \eta-1 / 2}\left[\operatorname{Cos}\left(\eta x^{2 \gamma / \eta}+\alpha\right)\{A\right.\left.+O\left(x^{-4 \gamma / \eta}\right)\right\} \\
&\left.+\operatorname{Sin}\left(\eta x^{2 \gamma / \eta}+\alpha\right) O\left(x^{-2 \gamma / \eta}\right)\right] \\
&+\sum_{j=1}^{p} x^{-2 \gamma\left(1-a_{j}\right)}\left\{E_{j}+O\left(x^{-2 \gamma}\right)\right\}
\end{aligned}
$$

where $\eta=2(n-p)=2(m-q), \alpha, A$ and $E_{j}$ are constants. Now writing $x=X^{\eta / 2 \gamma}$ and using the inequality

$$
\mathrm{Rl}\left(\frac{1}{2}-a_{j}\right)>\frac{1}{2 \eta}, \quad j=1, \cdots, p,
$$


of the enunciation of Theorem $1,(4.1)$ becomes

(4.2) $K\left(X^{\eta / 2 \gamma}\right) X^{\eta / 4 \gamma-1 / 2} \sim \operatorname{Cos}(\eta X+\alpha)\left\{A+O\left(X^{-1}\right)\right\}+O\left(X^{-\delta_{1}}\right)$,

where $\delta_{1}>1 / 2 \eta+1 / 2$. All the algebraical terms of (4.1) under the summation sign in (4.1) have been merged into the order term of (4.2). The left-hand side of (4.2) is nothing but a constant multiple of $K^{*}(x)$.

We have, similarly, for large real positive $x$,

$$
\begin{aligned}
& H(x) \sim x^{\gamma / \eta-1 / 2}\left[\operatorname { C o s } \left(\eta x^{2 \gamma / \eta}\right.\right.+\alpha)\left\{A+O\left(x^{-4 \gamma / \eta}\right)\right\} \\
&\left.+\operatorname{Sin}\left(\eta x^{2 \gamma / \eta}+\alpha\right) O\left(x^{-2 \gamma / \eta}\right)\right] \\
&+\sum_{j=1}^{q} x^{-2 \gamma\left(1+b_{j}\right)}\left\{E_{j}^{\prime}+O\left(x^{-2 \gamma}\right)\right\}
\end{aligned}
$$

and on writing $x=X^{\eta / 2 \gamma}$ and using

$$
\mathrm{Rl}\left(\frac{1}{2}+b_{j}\right)>\frac{1}{2 \eta}, \quad j=1, \cdots, q,
$$

of the enunciation of Theorem 1, (4.3) becomes

$$
\text { (4.4) } H\left(X^{\eta / 2 \gamma}\right) X^{\eta / 4 \gamma-1 / 2} \sim \operatorname{Cos}(\eta X+\alpha)\left\{A+O\left(X^{-1}\right)\right\}+O\left(X^{-\delta_{2}}\right) \text {, }
$$

where $\delta_{2}>1 / 2 \eta+1 / 2$.

5. Here we prove a theorem concerning a discontinuous integral associated with $K(x)$ and $H(x)$.

THEOREM 2. Let

$$
H_{1}(x)=\int_{0}^{x} H(u) d u .
$$

If the conditions (i), (ii) and (iii) of Theorem 1 hold, then

$$
\int_{0}^{\infty} K(x u) \frac{H_{1}(u y)}{u} d u= \begin{cases}0, & x>y>0, \\ \frac{1}{2}, & x=y>0, \\ 1, & y>x>0 .\end{cases}
$$

Proof. In Theorem 1 , take $f(y)$ defined by

$$
f(y)= \begin{cases}1, & y<Y, \\ 0, & y>Y\end{cases}
$$

so that 


$$
\frac{1}{2}\{f(x+0)+f(x-0)\}= \begin{cases}1, & Y>x>0 \\ \frac{1}{2}, & x=Y>0 \\ 0, & x>Y>0 .\end{cases}
$$

We have

$$
\int_{0}^{\infty} H(u y) f(y) d y=\int_{0}^{Y} H(u y) d y=\frac{H_{1}(u Y)}{u} .
$$

(5.1) then follows from Theorem 1.

6. Taking $p=q, m=n$ and

$$
\begin{aligned}
& a_{j}+b_{j}=0, \quad j=1, \cdots, p, \\
& c_{j}+d_{j}=0, \quad j=1, \cdots, m,
\end{aligned}
$$

we notice that

(6.1) $K(x)=H(x)=2 \gamma x^{\gamma-1 / 2} G_{2 p, 2 m}^{m, p}\left(x^{2 \gamma} \mid \begin{array}{l}a_{1}, \cdots, a_{p},-a_{1}, \cdots,-a_{p} \\ c_{1}, \cdots, c_{m},-c_{1}, \cdots,-c_{m}\end{array}\right)$

which is a symmetrical Fourier kernel obtained formally by the author in an earlier paper [6, p. 298]. This symmetrical Fourier kernel has been studied in detail by Fox [4, p. 396, Equation (7)].

\section{REFERENCES}

1. G. H. Hardy and E. C. Titchmarsh, $A$ class of Fourier kernels, Proc. London Math. Soc. 35 (1933), 116-155.

2. Roop Narain, The G-functions as unsymmetrical Fourier kernels. I, Proc. Amer. Math. Soc. 13 (1962), 950-959.

3. Bateman Manuscript Project, Higher transcendental functions, Vol. 1, McGrawHill, New York, 1953.

4. C. Fox, The $G$ and $H$ functions as symmetrical Fourier kernels, Trans. Amer. Math. Soc. 98 (1961), 395-429.

5. C. S. Meijer, On the G-function, Proc. Nedrl. Akad. Wetensch. 49 (1946), 227-237, 344-356, 457-469, 632-641, 765-772, 936-943, 1062-1072, 1165-1175.

6. Roop Narain, A Fourier kernel, Math. Z. 70 (1959), 297-299.

WASHINGTON UNIVERSITY 